\title{
34 \\ Specifying Relations between Research and the Design of Human-Computer Interactions
}

\author{
John Long \\ Ergonomics \& HCI Unit, University College London, UK \\ j.long@ucl.ac.uk
}

\begin{abstract}
This paper argues the need for more effective: human-computer interactions; design of such interactions; and research to support such design. One contribution to more effective research would be the specification of relations between research and the design of human-computer interactions. The aim of this paper is to propose such a specification. Meeting this specification renders HCI knowledge coherent, complete and 'fit-fordesign-purpose'. The absence of such relations renders Cognitive Science knowledge not coherent, complete nor 'fitfor-design-purpose' (as opposed to 'fit-for-understanding-purpose'). The paper concludes that specification of relations is required for more effective research support for the design of human-computer interactions.
\end{abstract}

KEY WORDS: research; design; human-computer interactions; effectiveness

\section{INTRODUCTION}

\subsection{Human-Computer Interactions}

Computing technology continues its inexorable advance. Computer applications pervade the home, the factory and the office. Computer developments continue to extend the limits of the technology - from novel hardware, such as speech recognisers, to novel software, such as spreadsheets. Novel complete technologies have been developed, such as integrated broadband communication and virtual reality (Long, 1991). Once the requirement was the computer and 'getting it to work at all', that is more effective computers. Now the main requirement might be said to be human-computer interaction, and 'getting the computer to work for us effectively', that is more effective human-computer interactions (Long and Dowell, 1996).

\subsection{Design}

Design of human-computer interactions has also continued to advance. Design includes both the specification of interactions and their subsequent evaluation, as well as the selection and training of users. Initially, the design of such interactions was a craft, which resided in the practices of programmers, designers, and user managers (Long and Dowell, 1989). Subsequently, attempts have been made to change these design processes by the behavioural sciences, such as Psychology and Sociology (also Cognitive Science - see Section 3), and the computing sciences, such as Software Engineering (Long, 1987). More recently, Human-Computer Interaction (HCI) research has also attempted to contribute to these design practices. Taken together, such design practices have achieved some notable successes. However, the success can also be viewed as limited. Notable failures have also been associated with such practices.

This view of current interaction design as being limited is reinforced, if the design practices are considered in terms of the 'capability maturity' model (Paulk, Curtis, Chrissis and Weber, 1993). The model has five levels of maturity. It would be difficult to classify current design practices as higher than Level 3,

Human-Computer Interaction: INTERACT'97 S. Howard, J. Hammond \& G. Lindgaard (editors)

Published by Chapman \& Hall CIFIP 1997 
that is 'defined'. Without the specification of such practices (and their relationship to research), their process cannot rise to the maturer levels of 4 'managed' and 5 'optimised'. Indeed, many design practices, currently, would be unlikely to achieve even Level 2, that is 'repeatable', that is, from project to project. There is a need, then, for more effective design (Dowell and Long, 1989).

\subsection{Research}

Research has also continued to advance the acquisition and validation of new knowledge to support design. Technically, the behavioural sciences have acquired new knowledge concerning the phenomena of interactions. Technically, $\mathrm{HCI}$ research has acquired new knowledge concerning techniques, methods, and tools to support design. Institutionally, a number of initiatives have funded research to support design. Although much useful research was conducted under various initiatives, little is known about the effectiveness of such knowledge, to support design. What has been established is often negative. In addition, little of the research has been validated. As a result, little of the knowledge acquired has been incremented. Failure to increment constitutes a failure in progress. As a consequence, the potential for applying such knowledge has been limited (Long and Dowell, 1996). There is a need, then, for more effective research.

\subsection{Specifying Relations}

More effective research for design would result in more effective human-computer interactions. Such research would require the validation of new knowledge. Thus would knowledge be incremented, and so progress made. However, such incrementation would only be effective, if the new knowledge were 'fit-for-designpurpose', that is to support design practices. The criterion here is relevance. If research is not relevant to design, the application of such knowledge could only be by trial and error. A pre-requisite for more effective research is the specification of relations between research and design. Specification of these relations would constitute one contribution to more effective research. Only if such relations are specified, and subsequently implemented, will new research knowledge be incrementable with respect to its effectiveness. Only then will validation of knowledge result in more effective human-computer interactions. The specification of such relations is the aim of this paper.

\section{SPECIFYING RELATIONS}

\subsection{Pre-requisites for Specifying Relations}

Informally, research should support design. Relations, then, might be identified and enumerated. Such a scheme, however, is inadequate. First, 'research' is a more general expression than 'designing humancomputer interactions'. Second, 'designing humancomputer interactions' is too rudimentary an expression to provide support for research. An expression is required, which is more coherent, complete and 'fit-forresearch-purpose'. These requirements indicate the following pre-requisites.

The first pre-requisite is a framework for disciplines. Such a framework should generalise design of humancomputer interactions, so that it can be expressed at the same high level as that of research. Such a framework could then be used to model, and so to specify, relations between $\mathrm{HCI}$ research and design. The second prerequisite is a framework for the $\mathrm{HCI}$ general design problem. The framework must be more coherent, complete and 'fit-for-research-purpose' than 'design of human-computer interactions'. However, it must also be expressed at a high enough level to support the modelling of alternative lower level descriptions of design. The model would specify the relations within the scope of the design to support research. The third pre-requisite is a framework for validation. The framework should be expressed at a high enough level to model both research (characterised as a discipline), and design of human-computer interactions (characterised as the $\mathrm{HCI}$ general design problem). Together, resulting models would, thus, embody, and so specify, the relations between research and design to support validation (see Figure 1). Meeting these pre-requisites would support more effective design.

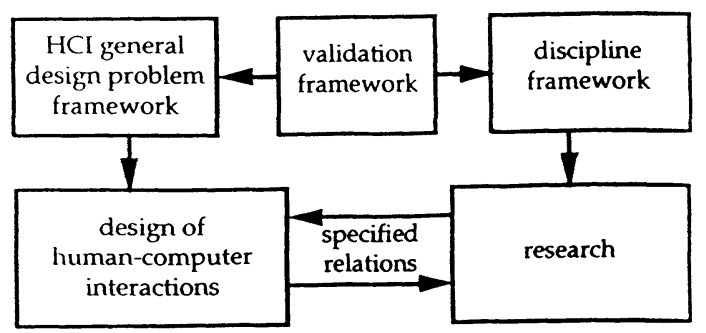

Figure 1. Frameworks to specify the relations between research and design of human-computer interactions to support the validation of knowledge. 
The discipline framework proposed here to model relations between $\mathrm{HCI}$ research and design of humancomputer interactions is taken from Long and Dowell (1989). A discipline (concepts are highlighted on first introduction) is assumed to possess three characteristics: knowledge; practices; and a general problem. Research, by its research practices, acquires knowledge. Knowledge supports practices solving the general discipline problem. Disciplines are, thus, distinguished by the general problem which they address. General discipline problems, however, also have a scope. Decomposition of a general problem, with respect to its scope, exposes general problems of particular scope. This decomposition allows the further division of disciplines into sub-disciplines. A discipline can thus be defined as: 'the acquisition by research practices, and use of knowledge to support practices, seeking solutions to a general problem having a particular scope' (see Figure 3).

\subsubsection{General Design Problem of HCI}

The HCI general design problem framework, proposed here to model alternative descriptions of design of human-computer interactions, is taken from Dowell and Long (1989). A domain of application of an HCI worksystem is where work originates, is performed, and has its consequences. Goals express a requirement for work. Goals are allocated to worksystems by organisations. A domain is distinct from, and delimits, a worksystem. A worksystem comprises at least two separate, but interacting, subsystems - namely human(s) and computer(s). Humans interact with computers to perform effective work. Effectiveness expresses how well the worksystem performs the work. The HCI general design problem, thus, can be expressed as: 'design humans interacting with computers to perform effective work'.

\subsubsection{Validation Framework}

The validation framework proposed here was used by Dowell and Long (1989) and Long and Dowell (1989). However, it is quite standard. Validation involves four processes: conceptualisation; operationalisation; test; and generalisation, and so four changes in the resulting products. Conceptualisation involves specified, and so explicit, representation. Operationalisation involves the mapping of concepts onto observables and eventually metrics. Test involves the evaluation of the assertions implicated in the concepts, which have been operationalised. Lastly, generalisation involves the abstraction and generification of the outcomes of the tests. The framework can be applied generally to knowledge, problems, etc. which are validated, if conceptualised; operationalised; tested; and generalised. Validation is required to ensure knowledge, problems, etc. are 'fit-for-purpose'. The pre-requisites for specifying the relations between research and the design of human-computer interactions have now been met. It remains to apply the frameworks to specify those relations.

\subsection{Specified Relations}

To specify the relations between research and the design of human-computer interactions, it is necessary to specify: the relations between $\mathrm{HCI}$ research and the $\mathrm{HCI}$ general design problem; and the relations within the particular scope of HCI to support HCI research, both with respect to the validation of knowledge, and so more effective design.

\subsubsection{HCI Research and the HCI general design problem}

The discipline framework and validation framework proposed earlier are now used to model the relations between $\mathrm{HCI}$ research and the $\mathrm{HCI}$ general design problem. Following Long and Dowell (1989) (see Section 2.1.1) the specification of the particular scope of $\mathrm{HCI}$ is: 'humans interacting with computers to perform effective work'. Given this particular scope of $\mathrm{HCI}$, its general problem can now be specified as: 'the design of humans interacting with computers to perform effective work'. Given this HCI general problem, its practices can now be specified as: 'diagnosis (of work (in)effectiveness) and prescription (of humans interacting with computers)'. Such practices solve the general design problem of humans interacting with computers to perform effective work. HCI knowledge supports HCI practices to solve this general design problem. Research, as supported by its practices, can now be specified as: 'the acquisition and validation of $\mathrm{HCI}$ knowledge'. Given HCI research, the validation of its knowledge can be specified as: 'the conceptualisation; operationalisation; test; and generalisation of its $\mathrm{HCI}$ knowledge'. Hence, a coherent and complete model of the discipline of $\mathrm{HCI}$ can be specified as: 'research supporting the acquisition and validation by research practices, as: conceptualisation; operationalisation; test; and generalisation, of $\mathrm{HCI}$ knowledge to support $\mathrm{HCI}$ practices of diagnosis and prescription, to solve the general $\mathrm{HCI}$ design problem of humans interacting with computers to perform effective work' (see right-hand panel of Figure 2). This specification relates research to design of human-computer interactions. 


\subsubsection{Particular scope of HCI to support HCI research}

The HCI general design problem framework and the validation framework are now used to model the relations within the particular scope of $\mathrm{HCI}$, to support $\mathrm{HCI}$ research. It might, of course, be thought that such specification exists - 'the design of humans interacting with computers to perform effective work'. However, this expression is not adequate to support research. Concerning the validation of HCI knowledge, the expression is insufficiently conceptualised to support: operationalisation, test and generalisation. Concerning the application of $\mathrm{HCI}$ knowledge, that is $\mathrm{HCI}$ practices, the expression is insufficiently conceptualised to support design, as diagnosis and prescription. A more completely conceptualised definition is thus required.

Following Dowell and Long (1989) (see Section 2.1.2) to model the general design problem of $\mathrm{HCI}$, it is necessary to specify: work; the worksystem, which performs the work; the goals of the worksystem; and the effectiveness with which the worksystem achieves its goals. The particular scope of the HCI general design problem is: 'humans interacting with computers to perform effective work'. Dowell and Long (1989) have proposed a conceptually more complete specification of $\mathrm{HCI}$ design as a general problem.

In the conception. a domain of application (of an HCI worksystem) is where work originates, is performed. and has its consequences. It comprises one or more objects, constituted of attributes, which have values. Goals express a requirement for change in the value of these attributes, and goals are allocated to worksystems by organisations. A domain is distinct from, and delimits. a worksystem. A worksystem comprises at least two separate, but interacting, subsystems - of human behaviours interacting with computer behaviours. These behaviours are supported by mutually exclusive human structures and computer structures, and are executed to perform work effectively. Effectiveness is expressed by the concept of performance, that is, how well a worksystem achieves its goals - that is, task quality, and the system costs that are incurred in so doing. Costs are incurred by both the human and the computer and are structural and behavioural.

Given this conception of the particular scope of $\mathrm{HCl}$, the validation of its general design problem (and solution) can now be specified as: 'the conceptualisation; operationalisation; test; and generalisation of the $\mathrm{HCI}$ general design problem (and solution)' (see left-hand panel of Figure 2).
The particular scope of the HCI discipline is the design of 'humans interacting with computers to perform effective work'. More completely, its particular scope would be the design of behaviours constituting a worksystem $\{\mathrm{S}\}$, whose actual performance $\left(\mathrm{P}_{\mathrm{A}}\right)$ conformed with some desired performance $\left(\mathrm{P}_{\mathrm{D}}\right)$. The design of $\{S\}$ would require the design of human behaviours $\{\mathrm{H}\}$ interacting with computer behaviours $\{C\}$. Hence, conception of the general HCI design problem would be: design $\{\mathrm{H}\}$ and $\{\mathrm{C}\}$,

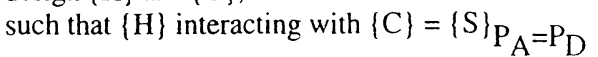
where $P_{D}=f n\left\{Q_{D}, K_{D}\right\}$.

$\mathrm{QD}_{\mathrm{D}}$ expresses the desired quality of work for the domain of application.

$\mathrm{K}_{\mathrm{D}}$ expresses acceptable costs incurred by the worksystem (both human and computer).

Design here should be understood to include both the specification of interactive worksystem behaviours and the evaluation of actual and desired performance. Design also includes: the physical and organisational environment; and selection and training of humans. to the extent that these factors determine interactive worksystem behaviours, and so actual and desired performance.

This model of the $\mathrm{HCI}$ general design problem specifies the relations within the particular scope of $\mathrm{HCl}$ to support research. Thus, according to this general design problem model, research is only 'fit-for-designpurpose' if it meets these specifications, that is, acquires and validates knowledge which supports the solution of HCI general design problems, so expressed. The specification of relations within the particular scope of $\mathrm{HCI}$, to support HCI research is now complete.

\subsubsection{HCI research and the HCI general design problem and particular scope of $\mathrm{HCI}$ to support HCI research}

Specified relations between $\mathrm{HCI}$ research and the $\mathrm{HCI}$ general design problem were modelled in Section 2.2.1. Specified relations within the particular scope of HCI to support research were modelled in Section 2.2.2. To specify the relations together, it suffices to integrate the two earlier sets of specified relations into a single model (see Figure 2].

Thus, according to these models of the $\mathrm{HCI}$ discipline and the $\mathrm{HCI}$ general design problem, research is only 'fit-for-design-purpose', if it meets these specifications, that is acquires and validates knowledge which supports the solution of the $\mathrm{HCI}$ general design problem, so expressed. The specification of relations is 


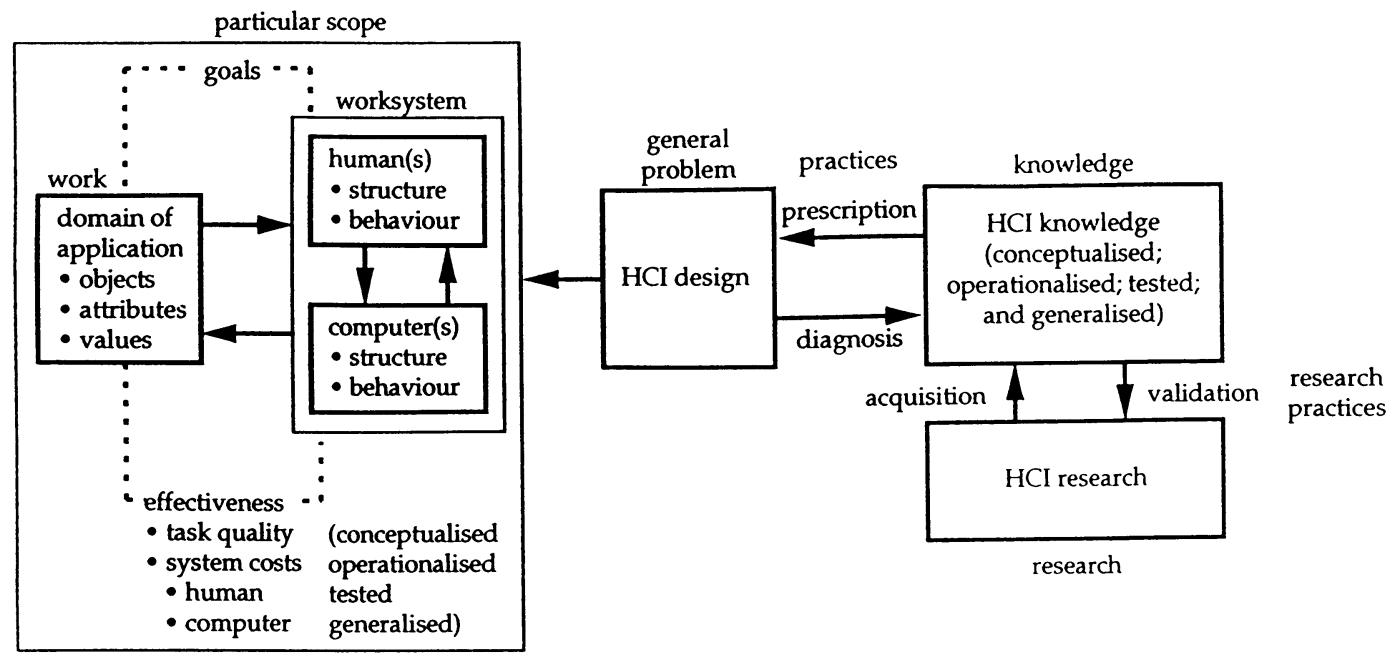

Figure 2. Model of the $\mathrm{HCI}$ discipline and model of the $\mathrm{HCI}$ general design problem specifying the relations between $\mathrm{HCI}$ research and the $\mathrm{HCI}$ general design problem, and within the particular scope of HCI, to support $\mathrm{HCI}$ research.

now considered to be complete. Taken together, these relations constitute one possible specification of the relations between research and design of humancomputer interactions.

\section{OTHER RESEARCH}

\subsection{Other research and HCI research}

It has been proposed how relations can be specified between research and the design of human-computer interactions. The question can now be posed, as to what relations obtain between other research, $\mathrm{HCI}$ research and the design of human-computer interactions. In particular, the concern is with research not conducted to support the design of human-computer interactions. The example selected here is Cognitive Science. Note that Cognitive Science is not unique. Similar relations, proposed later, could similarly be suggested for: Sociology; Ethnomethodology; Ethnography etc. The same frameworks and models used earlier will also be used here.

\subsection{Specified relations with} Cognitive Science research

Cognitive Science generally seeks to understand knowledge-based behaviours. Such behaviours include: perceiving; categorising; thinking; reasoning; communicating; problem-solving, etc. Cognitive Science might be defined non-controversially as: 'understanding the general principles underlying natural and artificial forms of intelligence'.

\subsubsection{Specified relations between Cognitive} Science Research and the Cognitive Science general understanding problem.

To specify the relations between Cognitive Science research and understanding natural and artificial forms of intelligence, it is necessary to model the Cognitive Science discipline. Given the particular scope of Cognitive Science, as natural and artificial forms of intelligence, its general problem can be specified as: 'the understanding of natural and artificial forms of intelligence' (understanding being the general problem of science as a discipline). Given this general problem, the understanding practices can be specified as: 'explanation and prediction'. Cognitive Science knowledge supports such practices. Cognitive Science research practices acquire and validate such knowledge as: conceptualised; operationalised; tested; and generalised (see right-hand panel of Figure 3). (Compare with the $\mathrm{HCI}$ model in Figure 2.)
3.2.2. Particular scope of Cognitive Science to support Cognitive Science Research
For present purposes, the particular scope of Cognitive Science, as natural and artificial forms of intelligence, is assumed to include at least: natural, that is human, intelligence and behaviour; artificial, that is, artefact 


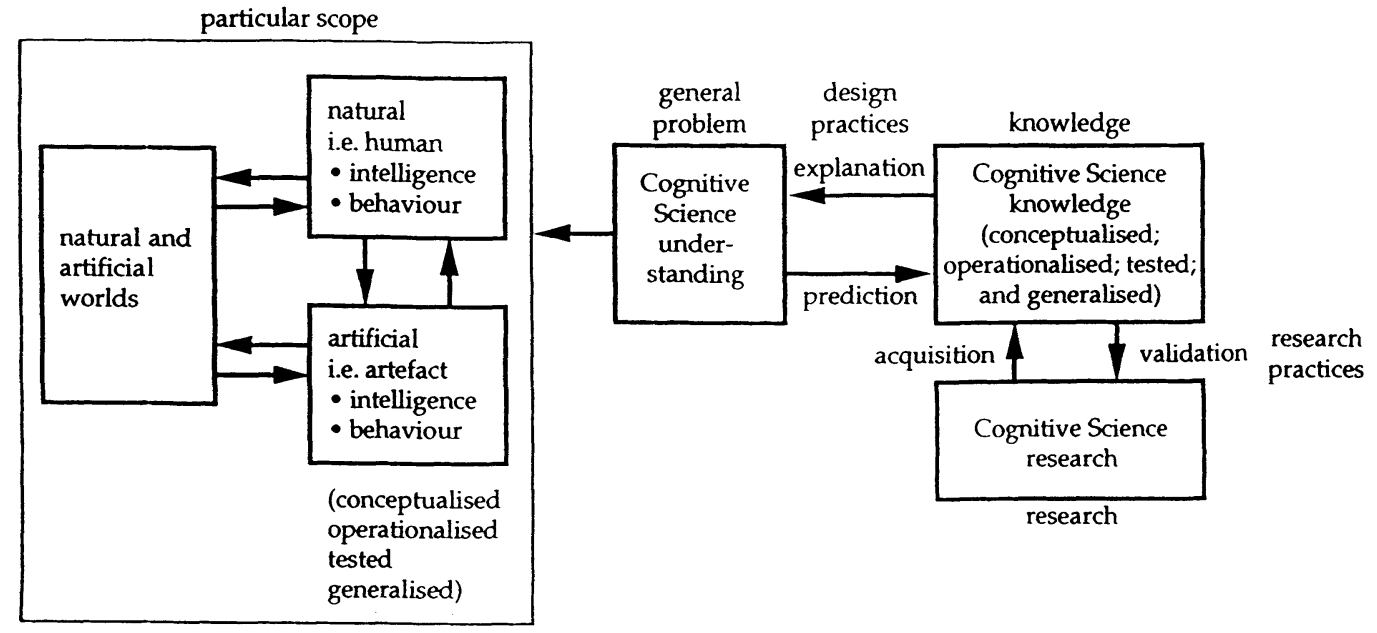

Figure 3. Model of the Cognitive Science discipline and model of the Cognitive Science general understanding problem specifying the relations between Cognitive Science research and the Cognitive Science general understanding problem, and within the particular scope of Cognitive Science, to support Cognitive Science research.

intelligence and behaviour; and natural and artificial worlds. Humans and artefacts are assumed to interact with one another, and with the world. Cognitive Science secks to understand the: intelligence; behaviour; and interactions (see left-hand panel of Figure 3). (Compare with the HCI model in Figure 2.)

\subsubsection{Cognitive Science discipline and the Cognitive Science general understanding problem}

Specified relations between Cognitive Science research and the Cognitive Science general understanding problem are modelled in Section 3.2.1. Specified relations within the particular scope of Cognitive Science, to support Cognitive Science research are modelled in Section 3.2.2. To specify the relations between the Cognitive Science discipline and the Cognitive S̄cience general understanding problem, it suffices to integrate the two earlier sets of specified relations into a single model (see Figure 3 ).

Thus, according to these models of the Cognitive Science discipline and the general understanding problem, research is only coherent, complete and 'fit-forunderstanding-purpose', if it meets these requirements. That is, it acquires and validates knowledge, which supports the solution of the Cognitive Science general problem, so expressed. The claim is the same as made earlier for $\mathrm{HCl}$. Knowledge produced by Cognitive Science research, and intended to support understanding, which embodies these relations, is more effective, than knowledge not embodying such relations.

\subsection{Cognitive Science Research and Design}

The question posed earlier can now be addressed. What relations obtain between other research, in this case Cognitive Science, and the design of humancomputer interactions? An assessment of possible relations can be made by comparing the relations within Cognitive Science (shown in Figure 3), with the relations within $\mathrm{HCI}$ (shown in Figure 2). Inspection of these figures shows there to be no specified relations between Cognitive Science research and the design of human-computer interactions. For example, Cognitive Science understanding practices have no specified relations with the diagnosis of general design problems and the prescription of general design solutions. The Cognitive Science general understanding problem has no specified relations with the performance (of worksystems). Thus, there are no specified relations between Cognitive Science research and the design of human-computer interactions. Cognitive Science research is, therefore, not coherent, complete, nor 'fitfor-design-purpose'. 


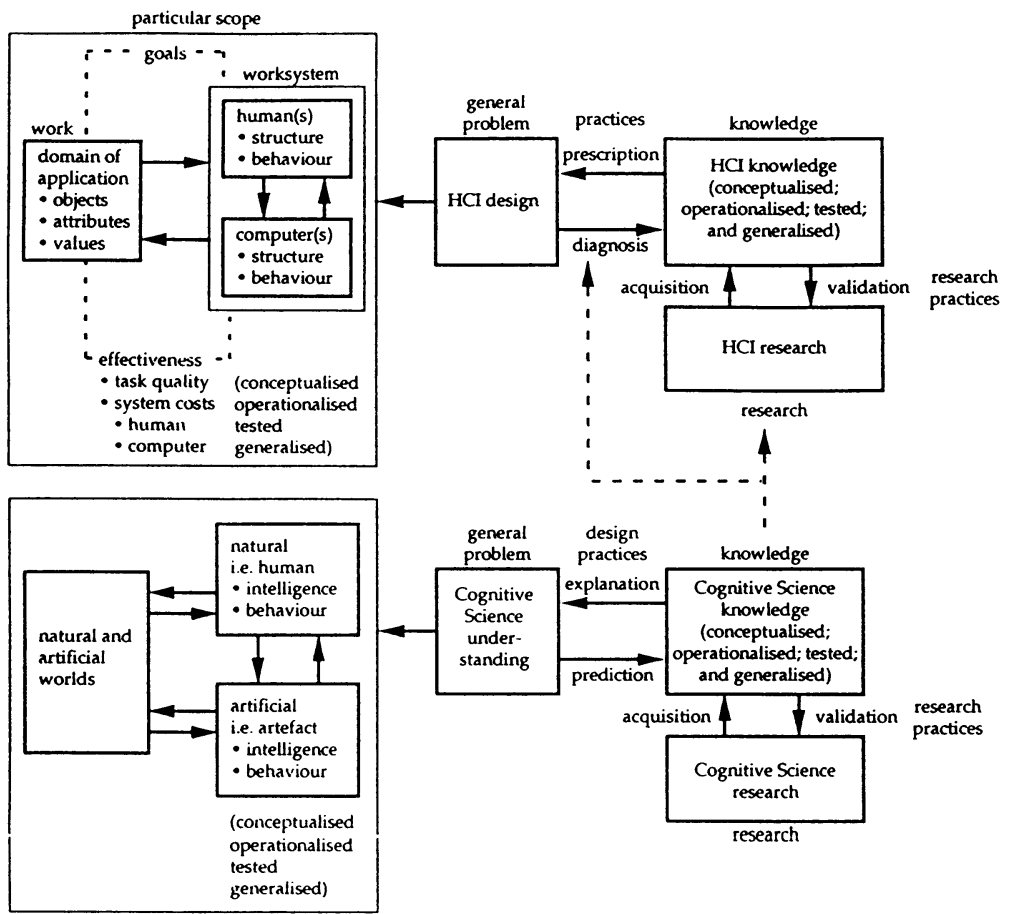

Figure 4. A model showing the unspecified relations (as dotted lines) between the $\mathrm{HCI}$ discipline and the $\mathrm{HCI}$ general design problem (Figure 2) and the Cognitive Science discipline and the Cognitive Science general understanding problem (Figure 3 ).
Note that a comparable conclusion must necessarily be drawn concerning the relations between $\mathrm{HCI}$ research and understanding natural and artificial forms of intelligence. Inspection of Figures 2 and 3 shows there to be no specified relations between $\mathrm{HCI}$ research and the understanding of natural and artificial forms of intelligence. For example, HCI design practices have no specified relations with the explanation of general understanding problems and the prediction of general understanding solutions. The HCI general design problem has no specified relations with (natural) intelligence, as expressed by humans.

It is, thus, concluded that research, not exhibiting the relations specified here with the design of humancomputer interactions, such as that undertaken by Cognitive Science, is not coherent, complete or 'fit-fordesign-purpose'.

\subsection{Unspecified Relations between Cognitive Science Research and Design}

It has been concluded that Cognitive Science research is not coherent, complete nor 'fit-for-design-purpose'. However, some researchers claim such a relationship. How might such a claim be understood with respect to the specified relations for Cognitive Science and HCI proposed here? This question can be addressed, if some relationship can be established between the $\mathrm{HCI}$ discipline and its general design problem (Figure 2) and the Cognitive Science discipline (Figure 3). The relevant models are shown in Figure 4 . The models suggest two ways in which Cognitive Science might contribute to design. First, Cognitive Science knowledge might contribute to $\mathrm{HCI}$ research practices of the acquisition and validation of $\mathrm{HCI}$ knowledge, such practices having specified relations with the design of human-computer interactions. Second, Cognitive Science knowledge might contribute to $\mathrm{HCI}$ practices of diagnosis and prescription, and so of design, such practices having specified relations with design.

However, in both cases, the relations between Cognitive Science knowledge and HCI research, and HCI practices are unspecified. Hence, any relations could only be implicit. Such implicit relations could only be realised via the explicit relations specified between $\mathrm{HCI}$ research and the design of human-computer interactions. Further, the realisation of such implicit relations could only have the status of a 'craft'. That is, any 'knowledge' of transforming the Cognitive Science knowledge base to support either $\mathrm{HCI}$ research or $\mathrm{HCI}$ practices, is likely to reside only in the experience and 
skill of the individual researcher or design practitioner. Thus, there would be no validation of the transformation with respect to its support for the design of humancomputer interactions. Cognitive Science knowledge, thus, is able to contribute to HCI design and research practices. However, such contributions can only be implicit, since unspecified. They cannot as such be known.

\subsection{Other Research, HCI Research and Design}

Readers might be interested to assess the relations between their own research (and that of others) and the design of human-computer interactions, in terms of the relations specified here. First, research can be assessed against the model of the $\mathrm{HCI}$ discipline and the model of the HCI general design problem (Figure 2). The research can be further assessed against the relations within the particular scope of HCI, to support HCI research (Figure 2). Second, research can be assessed against the model of the Cognitive Science discipline and the model of the Cognitive Science general understanding problem (Figure 3 ). The research can be further assessed against the relations within the particular scope of Cognitive Science, to support Cognitive Science research (Figure 3). Third, research can be assessed for unspecified relations between Cognitive Science research and the design of humancomputer interactions (Figure 4). However, if research is neither HCI nor Cognitive Science (for example, Ethnomethodology, etc), then, the frameworks proposed for disciplines, the HCI general design problem and for validation, must be used to construct models of the kind appearing in Figures 2 and 3 . Relations can then be specified. Assessment of the research can then be made against the models of specified relations.

\section{SUMMARY \& CONCLUSIONS}

This paper began by summarising current and past technical and institutional developments, as they concern the design of human-computer interactions and research. The paper argues the requirement for more effective: human-computer interactions; design of such interactions; and research support for such design. Greater effectiveness of interactions, practices and research will derive from the validation of new $\mathrm{HCI}$ knowledge, and the specification of relations between $\mathrm{HCI}$ research and the design of human-computer interactions, such that research is 'fit-for-designpurpose'. This paper attempts to specify such relations. It is suggested how research with unspecified relations to the design of human-computer interactions, such as
Cognitive Science, can only contribute implicitly to the design of human-computer interactions. Such implicit contributions can only be realised via the specified relations of $\mathrm{HCI}$.

In conclusion, if a serious attempt is to be made to improve the effectiveness of: human-computer interactions; the design of such interactions; and the research support for such design, there is a need to ensure coherence, completeness and the 'fitness-fordesign-purpose' of research to support the design of humans interacting with computers to perform work effectively, including validation for that purpose. All disciplines, which conduct research claiming to contribute to the design of human-computer interactions for effective work, need to specify the relations between their research and the design of human-computer interactions. Specifying relations between $\mathrm{HCI}$ research, and the $\mathrm{HCI}$ discipline, and within the design of human-computer interactions, expressed as the general HCI design problem, to support HCI research, as proposed in this paper, is one means of so doing.

\section{Acknowledgments}

I would like to acknowledge my debt to colleagues at the Ergonomics \& HCI Unit, UCL, and especially to John Dowell by whom or with whom all the important ideas in this paper have been developed.

\section{References}

Dowell, J. and Long, J. (1989). Towards a Conception for an Engineering Discipline of Human Factors. Ergonomics, 32 (11). 1513-1535.

Long, J. (1987). Cognitive Ergonomics and Human Computer Interaction. In Psychology at Work, P. Warr (ed). Harmondsworth, UK: Penguin.

Long, J.B. (1991). Human-Computer Interaction and the Information Technology Revolution, or Getting Computers to Work for Us Effectively. In British Gas White Paper series.

Long, J. and Dowell, J. (1989). Conceptions for the Discipline of HCI: Craft, Applied Science and Engineering. In A.Sutcliffe and L.Macaulay (eds), People and Computers IV, Proceedings of the Fifth Conference of the BCS HCI SIG. Cambridge, UK: CUP. 9-32.

Long, J. and Dowell, J. (1996). Cognitive Engineering or 'getting users and computers interacting to perform work effectively'. The Psy'chologist, 9 (7), 313-317.

Paulk, M.C., Curtis, B., Chrissis, M.B. and Weber, C.V. (1993). Capability Maturity Model, Version 1.1. IEEE Software, 18-27. 